\title{
An Infinite Lottery Paradox
}

\author{
John D. Norton \\ Department of History and Philosophy of Science \\ University of Pittsburgh \\ Pittsburgh PA 15260, USA \\ jdnorton@pitt.edu \\ and \\ Matthew W. Parker \\ Rotman Institute of Philosophy \\ University of Western Ontario \\ London, Ontario N6A 5B7, Canada \\ matthew.parker@uwo.edu
}

Forthcoming in Axiomathes, Special Issue Epistemologia 2022

\begin{abstract}
In a fair, infinite lottery, it is possible to conclude that drawing a number divisible by four is strictly less likely than drawing an even number; and, with apparently equal cogency, that drawing a number divisible by four is equally as likely as drawing an even number.
\end{abstract}

Keywords: additivity, cardinality, comparative probability, inductive logic, infinite lottery, probability

\section{Introduction}

A fair, infinite lottery selects without favor among a countable infinity of possible results, numbered $1,2,3, \ldots$. It is realized in a thought experiment by an imaginary machine that can 
select from an infinity of balls, numbered $1,2,3, \ldots$, such that none is favored. This thought experiment has long had a place in analyses of the foundations of probability. De Finetti (1972, $\S 5.17)$ used it to argue against the countable additivity of probability measures. Benci, Horsten and Wenmackers (2018) used it to argue for infinitesimal probabilities.

Subsequently, one of us (Norton, forthcoming), has argued that the infinite lottery thought experiment requires us to discard not just a countably additive probability measure, but even a finitely additive notion of chance. Norton's analysis depends on realizing the condition of selection without favor as the requirement that the likelihood or chance of some outcome depends only on the sizes of the set of balls favorable to it and the set of balls unfavorable to it. Norton's implementation, the other of the present authors (Parker, 2020) has objected, presupposes that chances are the values of a function over the outcome space. Parker has presented an alternative analysis, in terms of a comparative chance relation, that restores finite additivity (in a comparative form) and also makes every outcome set more likely than any of its proper subsets. (For a response, see Norton, forthcoming a, §11.)

This note reviews the arguments for each analysis and leaves the final decision as an open question. The two arguments depend on different notions of the comparison of sizes of infinite sets. Do we compare them better by cardinality or by set theoretic inclusion? Norton's analysis privileges the first over the second. Parker's analysis privileges the second over the first. 
The precise notion of chance ${ }^{1}$ invoked here will remain incompletely specified. The paradox is intended to be a motivation to discover which formal properties of a notion of chance allow the paradox to be addressed most satisfactorily.

\section{The Paradox}

A formal condition, 'label independence,' asserts that the chance of an outcome, specified as a set of numbers, is unaffected by any relabeling that merely permutes the numbers assigned to the balls. This condition expresses the fairness of the drawing since a permutation preserves the sizes of the sets, both favorable and unfavorable to the outcome. Those sizes are, by supposition, all that determines the outcome chances. For example, the outcome even is just the drawing of a ball with an even number; and odd is the drawing of an odd number. A permutation of the numbering merely switches the numbers on the sets of balls associated with the two outcomes according to $1 \leftrightarrow 2,3 \leftrightarrow 4,5 \leftrightarrow 6, \ldots$ It now follows that even and odd outcomes must have the same chance, since the outcome set originally labeled even has become odd under the relabeling, and conversely.

A second condition on the chances is also attractive. The outcome 'fours' is just the set of multiples of $4:\{4,8,12,16, \ldots\}$. It is a proper subset of even. Thus, whenever we have an outcome fours we have an outcome even, but not conversely. Hence the chance of fours should be strictly less than that of even. To strengthen this last conclusion, consider the outcome 'fours $2^{\prime}:\{4-2,8-2,12-2,16-2, \ldots\}=\{2,6,8,10,14, \ldots\}$. The outcomes fours and fours -2 are

\footnotetext{
1 'Chance' here is not intended to imply a notion of objective physical chance like those associated with propensity, frequency, or Humean best-systems accounts, nor do we exclude such interpretations by fiat. We adopt 'chance' as a convenient word distinct from 'probability,' for we do not assume the standard probability axioms.
} 
related by a permutation of labels and so have equal chances under label independence. The outcome even is partitioned into just these two outcomes, fours and fours -2 . Hence even and fours do not differ by an outcome set negligible on chancy scales, but by an outcome whose chance equals that of fours.

The analysis so far involves several individually plausible propositions: ${ }^{2}$

(1) Label independence. The chances of outcomes in a fair, infinite lottery are unchanged under a permutation of outcome labels.

(2) Containment. Outcome fours has strictly less chance than even.

(3) Completeness. It is possible to assign mutually comparable chances to all outcomes of a fair, infinite lottery.

The paradox is that these propositions are jointly inconsistent. For there is a permutation of the balls that takes those that were numbered even and maps them just to those numbered fours. The induced relabelings are:

$$
2 \rightarrow 4,4 \rightarrow 8,6 \rightarrow 12,8 \rightarrow 16 \ldots 1 \rightarrow 1,3 \rightarrow 2,5 \rightarrow 3,7 \rightarrow 5, \ldots
$$

Read these to say, 'The ball formerly numbered 4 is now relabeled 2,' etc. It now follows from label independence that if fours has chance $X$, then so does even. For they are each realized by the same ball drawings, but now just labeled differently.

The paradox can be escaped by denying at least one of the propositions above. The present authors differ, both from each other and from other authors, on which to deny.

\footnotetext{
2 This is not meant to be a complete analysis of all the implicit background assumptions in the finest possible granularity. It is just enough to set up our paradox.
} 


\section{The Probabilists' Escape}

Probabilistic approaches to infinite lotteries escape the paradox by denying Label Independence (1). It is well known that if each single outcome $\{1\},\{2\},\{3\}, \ldots$ has the same chance, then the probability axiom of countable additivity fails. Probabilists have often been willing to reject countable additivity in the case of an infinite lottery while still regarding finite additivity as essential to coherence (e.g., De Finetti 1972; Benci et al. 2018).

In imposing a finitely additive measure, probabilists are changing the problem posed. The defining characteristic of this version of the infinite lottery problem is that the chance of an outcome depends on the size of the outcome set and its complement; and only these sizes. A finitely additive measure violates the 'only' condition. For any set of balls that is both infinite and co-infinite, there is a numbering in which that set of balls is the even-numbered set. Once a designation of even is made and a specific probability $1 / 2$ is assigned to it, finite additivity implies that most other infinite, co-infinite sets cannot have that same probability. Which of all possible infinite co-infinite sets in the outcome space can be even and carry the same probability is determined by restricting the numberings to a subset of all possible numberings. The requirement of these preferred numberings adds structure to the problem in a way that violates the 'only' condition. For, both set sizes and preferred numberings are now required in order to determine the probabilistic chances of outcomes.

In short, denying Label Independence (1) does not address the problem posed here. We are not suggesting that cardinality and co-cardinality determine chances in other situations such as a game of darts with a continuous dartboard. But here, denying label independence does not solve the problem; it merely changes the problem posed. 


\section{The Cardinality Escape}

One of us (Norton) denies Containment (2) in order to preserve Completeness (3). We saw above that the distinctive characteristic of a fair, infinite lottery, label independence, requires that even and fours have equal chances (if they have chances at all). This result is only unwelcome, Norton claims, if one proceeds with intuitions tutored by finite sets. Among infinite sets, a set can have the same size (cardinality) as one of its proper subsets. Cardinalities of sets and their complements alone should determine the equality of chances.

What results is a novel account of the chances of outcomes of infinite lottery drawings that assigns a chance to all sets of outcomes. Completeness (3) is preserved. Finite sets of outcomes are assigned various, very small chances. Infinite sets that are co-finite are assigned various very high chances. The intermediate case-infinite sets of outcomes that are coinfinite - all have the same intermediate chance.

One might conjecture that this escape is untenable since, in repeated drawings, the outcome even should, with increasing chances, occur roughly twice as often as fours. However, the chance relations arising from label independence do not support this difference of frequencies. That is, $n$ outcomes of even among $N$ drawings has the same chance as $n$ outcomes of fours among $N$ drawings (for all $N$ and each $n, 0 \leq n \leq N$ ). Label independence ensures that the outcome fours has the same chance as even in a single draw, so neither is favored. By similar means, label independence ensures that repeating the drawings continues equally to favor equal frequencies of fours and even. While this outcome is unlike the corresponding result in familiar probability theory, it is only an unfamiliar result of an unfamiliar calculus. It is no more curious than the original result that fours and even have the same chance. (For details, see Norton, forthcoming.) 


\section{The Containment Escape}

The other of us (Parker) defends the alternative of denying Completeness (3). Perhaps we are not justified in supposing that all outcome sets can be mapped into one linearly ordered set of things called chances. Instead, we can define a partial relation of 'is at most as likely as' between outcome sets, a relation that satisfies label independence and yet makes fours less likely than even, so that both Label Independence (1) and Containment (2) hold. ${ }^{3}$ On this view, chances are still determined by the sizes of the favorable and unfavorable sets, but with two provisos: (i) 'Size' is understood in a sense that favors the Euclidean axiom, that the whole is greater than the part, over Cantor's criterion of 1-1 correspondence (cf. Mancosu 2009, Parker 2009), and (ii) chance is a partial two-place relation on events, rather than a total one-place function. Then chance relations are determined by size when the events are comparable, which is not always the case. Disjoint, countably infinite sets like even and $o d d$, for example, remain incomparable. We could even adopt a partial, relational notion of size, so that relative size (and incomparability thereof) always determines relative chance (and incomparability thereof).

The value of making fours less likely than even (Parker claims) is not merely to preserve intuitions tutored by finite sets. In one respect, it makes chance a better guide to decision making. Suppose you are offered a chance to make one of two bets at the same cost: that the outcome of our drawing will be in even or that it will be in fours. If in fact the outcome is in fours, it is also in even, so you win on either bet. If, however, the outcome is in even, it might

3 (1) and (2) are mutually consistent in this relational context, for here we cannot assert that the chance of fours is some specific value $X$ and then infer by label independence that the chance of even is also $X$. For a specific chance relation, and proof that it satisfies (1) and (2), see Author 2 2020. 
not be in fours. In fact, there are infinitely many such outcomes, forming the set fours -2 , which, on either view, is non-negligibly likely to occur. Hence it would be irrational to bet on fours when one could more safely bet on even for the same price. If fours and even are assigned equal chances, those chances do not tell us which bet to choose, whereas if fours is taken to be less likely than even, the chances capture the asymmetry of such betting scenarios and related decisions.

It is important that this notion of chance is not only partial, leaving some outcome sets incomparable, but also purely comparative. It will not do to have instead a monadic chance function over some proper subclass of the events. For then, label independence would force a poor outcome: either fours and even have the same chance, or no infinite, co-infinite set is assigned a chance at all. If even one such set is assigned a chance, then by label independence, they all are, and they are all assigned the same chance. A function that instead assigns chances only to finite and co-finite sets would fail to capture the feature that the infinite, co-infinite sets are intermediate in chance between the finite and co-finite ones. As well, it would not give even greater chance than fours. Therefore, a partial, comparative chance relation is here far preferable to a partial, monadic chance function, for it expresses much more.

To this one might reply, following Easwaran 2014, that a chance function need not express all the relative chances between proper subsets and supersets, for those are captured instead by the subset relations themselves. But that is just to admit that the chance function does not tell the whole story, nor, in this case, hardly any of it. It is only a superfluous representation of a small portion of the relative chances implied by the setup. Or so says Parker. 


\section{Conclusion}

How are we to choose between Containment (2) and Completeness (3)? We are at an impasse. The difference between our two views corresponds to two different ways of comparing the sizes of outcome sets and thus the chances these sizes dictate. ${ }^{4}$ Do we compare them by cardinalities and suffer the consequence that fours is as likely as even? Or do we compare them by the Euclidean axiom and suffer the consequence that the chances of many outcomes, such as even and $o d d$, are incomparable?

\section{Acknowledgement}

This work was partly supported by the John Templeton Foundation under grant \#61048.

\section{References}

Norton, J. D. forthcoming. "Infinite Lottery Machines," Ch. 13 in The Material Theory of Induction. BSPS Open. Ms at http://www.pitt.edu/ jdnorton/homepage/cv.html\#material_theory

Norton, J. D. forthcoming a. Author's responses. Studies in History and Philosophy of Science Part A. Online October 9, 2020 at https://www.sciencedirect.com/science/article/abs/pii/S0039368120301837

\footnotetext{
4 The paradox is thus related to Galileo's Paradox (Parker, 2009) but suggests further that one's conception of set size bears on what one should expect in this chance experiment.
} 
Parker, M. W. 2009. Philosophical method and Galileo’s paradox of infinity. In New Perspectives on Mathematical Practices, ed. B. van Kerkhove, 76-113. Singapore: World Scientific.

Parker, M. W. 2020. Comparative infinite lottery logic. Studies in History and Philosophy of Science Part A 84: 28-36.

Benci, V., L. Horsten and S. Wenmackers. 2018. Infinitesimal probabilities. British Journal for the Philosophy of Science 69: 509-552.

De Finetti, Bruno 1972. Probability, Induction and Statistics. London: John Wiley \& Sons.

Easwaran, K. 2014. Regularity and hyperreal credences. Philosophical Review 123: 1-41.

Mancosu, P. 2009. Measuring the size of infinite collections of natural numbers: Was Cantor's theory of infinite number inevitable? Review of Symbolic Logic 2: 612-646. 